\title{
An Introduction to Continuum Distorted Wave Theory
}

\author{
D. S. F. Crothers \\ Department of Applied Mathematics and Theoretical Physics, Queen's University Belfast, Belfast BT7 1NN, UK \\ Correspondence should be addressed to D.S. F. Crothers, d.crothers@qub.ac.uk
}

Received 11 November 2009; Revised 25 February 2010; Accepted 1 June 2010

Academic Editor: Roberto Daniel Rivarola

Copyright (C) 2010 D. S. F. Crothers. This is an open access article distributed under the Creative Commons Attribution License, which permits unrestricted use, distribution, and reproduction in any medium, provided the original work is properly cited.

The author gives an introduction to Continuum Distorted Wave theory, in the form of a brief review.

\section{Introduction}

Originally a continuum distorted wave (CDW) was a quantum mechanical Coulomb wave associated with the motion of an electron attached to an ion/atom relative to, and, while simultaneously in, the continuum of another ion [1].

The context was charge transfer. Subsequently this was generalized to the continuum of an electron [2], but not before it was generalized to ionization in an ion-atom collision [3]. Based on these latter two papers, Crothers and McCann [4] invented CDWEIS (eikonal initial state) which guarantees unitarity of the propagating initial state. Other later notations for the electron projectile case [2] are C3 and BBK. CDW-CDW is appropriate for high impact energies and small and medium impact parameters. CDW-EIS is appropriate for large impact parameters and lower energies.

One of the principal advantages of CDW theory lies in its Coulomb phases which guarantee the correct asymptotic/boundary behaviour [5-7].

Of course CDWEIS is intended to describe ionization in intermediate and high-energy collisions. At lower energies charge transfer is an important intermediate event.

The CDW theory is an on-shell theory and avoids artificial logarithmic potentials and spurious nonlocal operators [6]. The latter accordingly used generalized nonorthogonal coordinates (gnoc). If the electron has position coordinates $\underline{r}_{\mathrm{T}}$ and $\underline{r}_{P}$ with respect to the target and projectile atomic (or molecular) nuclei, respectively, then the net perturbation is the non-orthogonal kinetic energy $-\underline{\nabla}_{\underline{r}_{\mathrm{T}}} \cdot \underline{\nabla}_{\underline{r}_{P}}$.

Elastic-divergence-free CDW Neumann-Born series may be derived [8] and generalization to more than one electron is straight forward.
Rivarola and Fainstein [9] generalized the exact oneelectron formulation to the Roothan-Hartree-Fock treatment of effective one-electron problems. Jones and Madison [10] applied the formulation of Crothers [2] to actual electron-atom collisions.

The independent-event model was introduced in 1987 by Crothers and McCarroll [11]. This should not be mistaken for the more familiar independent-electron model. The independent-event model is possible because an alternative to the all-encompassing wave treatment is the impact parameter treatment [12]. It is a particular case of the timedependent Schroedinger (or Hartree-Fock) equation.

As described by Crothers [13], Crothers and Holt [14], and McDowell and Coleman [15], the transition between the full-wave and impact-parameter treatments is facilitated by a two-dimensional Fourier transform, the two dimensions being orthogonal to the relative velocity. This leads to the familiar eikonal representation only in the case of (total) cross-sections with atomic spherical symmetry.

A further example of the power of the independent-event model is in the description of Thomas double scattering electron-capture which is well-described as a second-order CDW event [16]: CDW2, third-order being negligible and given the necessary small impact parameters for large angle scattering. In the case of capture and/or excitation, coupled equations may be formulated variationally [17].

Generalized (G) CDWEIS was introduced by Crothers et al. [18] in which a complete set of magnetically quantized CDWs was introduced successfully. So, strong Coulomb interaction, event-correlation, gauge and Galilean invariance, detailed balance, intermediate continuum coupling, stationary or nonstationary: electron capture, ionization, transfer-ionization and other double events are all described. 
For instance, Pedlow et al. [19, 20] compared GCDWEIS, on the whole favourably with experimental findings for fully differential cross sections for ionization in the four-body 3.6 $\mathrm{MeV}$ amu ${ }^{(-1)}: \mathrm{Au}^{[24+, 53+]}+$ He collisions.

In addition Crothers $[21,22]$ generalized CDWEIS to (relativistic R) CDW-EIS, including such events as radiative capture, $\mathrm{X}$-ray emission, and pair production at relativistic impact velocities/energies.

Lest I forget, the major differences between heavyparticle and light-particle collisions (notably the projectile) are the consequences of electron-translation factors (ETF): thus factors like $\exp ( \pm \underline{i v} \cdot \underline{r})$ where $\underline{v}$ is the relative heavyparticle velocity and $\underline{r}$ is the electron coordinate relative to say the heavy-particle centre of mass.

For any appreciable velocity $\underline{v}$, this ETF is nonperturbative and has an effect well beyond the dipole $\underline{v} \cdot \underline{r}$. This is why R-matrix theory [23] and Density Functional Theory (Wikipedia) [24] have limited applications in heavyparticle collisions.

For those who wish to develop multibody CDW applications I would suggest Messiah [25]: Ch IX, para 13, Crothers and Dubé [6], Pedlow et al. [19, 20], and the works of Belkić, Rivarola, and Fainstein.

In 1991 I had the honour of giving a CDW talk at a meeting in Durham to mark the retirement of Professor Brian $\mathrm{H}$ Bransden. Also attending was Dr Ian Cheshire and he was truly amazed at the 27-year progress in CDW and its applications.

Another 19 years have passed!

\section{Further Historical Observations}

A scattering theory for a three-particle system was given by Faddeev [26] before Cheshire [1] and extended by Lovelace [27]. However, this theory is designed to describe nuclearphysics reactions with short-range interactions. As emphasised by Mishima et al. [28], these are off-shell theories with disconnected kernels and freely propagating third particles, for long-range Coulomb dominated collisions, unlike CDW theory in which all three particles are connected by the nonorthogonal kinetic-energy dipole-dipole operator.

Dodd and Greider [29] used the limit of small $m_{e} / M$ (where $m_{e}$ is the electron mass and $M$ is the typical mass of a nucleus) in a heavy-particle collision using the FaddeevLovelace formalism. However applying perturbation theory to a theory which has the above mentioned flaws, does not appear to be fortunate [30]. Cheshire's theory does not have these flaws, being on-shell a priori. And of course disconnected kernels are associated with elastic divergences.

A similar problem results from the strong-potential Born approximation [31]. Target (or projectile) CDW theory is better founded, that is, in the limit of high target (or projectile) charge, compared with the projectile (or target) charge [32]. A correct Dodd-Greider on-shell treatment is given [8] on the understanding that the bra(c)ket contains heavy-particle plane waves $e^{-i \underline{k}_{f} \cdot \underline{R}}$ and $e^{i \underline{k}_{i} \cdot \underline{R}}$, respectively, where $\underline{R}=\underline{r}_{T}-\underline{r}_{P}$ in Section 1 notation, and by Belkic et al. [33]. The latter reviews Belkić et al. [34-36] and CDW4B including Born (B), boundary continuum intermediate states (BCIS), Born Initial State (BIS), Coulomb-Born (CB), Oppenheimer-Brinkman-Kramers (OBK), classical trajectory Monte Carlo (CTMC), Distorted-wave Born (DWB) and four-body corrected First-Born (CB1-4B), observing that $\mathrm{CDW}-4 \mathrm{~B}$ overestimates experimental data at lower impact energies. Any theory involving B for Born (as against B for body) involves disconnected off-shell kernels and does not satisfy the long-range boundary conditions.

Regarding relativistic CDW (RCDW) one may refer to Crothers [21], particularly Chapters 6 and 7. On p299 of the latter book, nine references to Deco and Rivarola are listed together with three other Deco references. Readers may peruse these at their leisure. Nonrelativistic models [33] concern single and double electron capture, electron ionization, transfer excitation, and transfer ionization. Relativistic models [21] concern capture, pair production, and ionization at both relativistic and ultrarelativistic impact energies.

\section{Conclusions}

The method of Continuum Distorted Waves is a powerful, robust theory to describe heavy-particle collisions and even light-particle collisions. The independent-event model in the impact parameter treatment remains the best way forward to describe mechanisms quantitatively, while the use of complete magnetic-degenerate basis sets remains to be further exploited not least for multiply differential cross sections.

\section{Acknowledgments}

The author acknowledges, as Professor Emeritus and Honorary Visiting Senior Research Fellow, an office in the David Bates Building, QUB, and access to electronic journal resources.

\section{References}

[1] I. M. Cheshire, "Continuum distorted wave approximation; resonant charge transfer by fast protons in atomic hydrogen," Proceedings of the Physical Society, vol. 84, no. 1, pp. 89-98, 1964.

[2] D. S. F. Crothers, "Refined orthogonal variational treatment of continuum distorted waves," Journal of Physics B, vol. 15, no. 13, pp. 2061-2074, 1982.

[3] D. Z. Belkić, "A quantum theory of ionisation in fast collisions between ions and atomic systems," Journal of Physics B, vol. 11, no. 20, pp. 3529-3552, 1978.

[4] D. S. F. Crothers and J. F. McCann, "Ionisation of atoms by ion impact," Journal of Physics B, vol. 16, no. 17, pp. 3229-3242, 1983.

[5] L. Rosenberg, "Variational methods in charged-particle collision theory," Physical Review D, vol. 8, no. 6, pp. 1833-1843, 1973, P. J. Redmond, unpublished.

[6] D. S. F. Crothers and L. J. Dubé, "Continuum distorted wave methods in ion-atom collisions," Advances in Atomic, Molecular, and Optical Physics, vol. 30, pp. 287-337, 1992.

[7] J. D. Dollard, "Asymptotic convergence and the Coulomb interaction," Journal of Mathematical Physics, vol. 5, no. 6, pp. 729-738, 1964. 
[8] D. S. F. Crothers, "Strong coulomb interactions in atomic physics," Nuclear Instruments and Methods in Physics Research, $B$, vol. 27, no. 4, pp. 555-564, 1987.

[9] R. D. Rivarola and P. D. Fainstein, "Application of distorted wave models for electron emission in ion-atom collisions," Nuclear Instruments and Methods in Physics Research, B, vol. 24-25, no. 1, pp. 240-242, 1987.

[10] S. Jones and D. H. Madison, "Evidence of initial-state twocenter effects for (e, 2e) reactions," Physical Review Letters, vol. 81, no. 14, pp. 2886-2889, 1998.

[11] D. S. F. Crothers and R. McCarroll, "Correlated continuum distorted-wave resonant double electron capture in $\mathrm{He}^{2+}-\mathrm{He}$ collisions," Journal of Physics B, vol. 20, no. 12, pp. 2835-2842, 1987.

[12] N. F. Mott, "On the theory of excitation by collision with heavy particles," Mathematical Proceedings of the Cambridge Philosophical Society, vol. 27, p. 553, 1931.

[13] D. S. F. Crothers, Theoretical studies of inelastic atomic collisions, Ph.D. thesis, Queen's University Belfast, Belfast, UK, 1966.

[14] D. S. F. Crothers and A. R. Holt, "The first Born approximation for collisions between heavy particles," Proceedings of the Physical Society, vol. 88, no. 1, pp. 75-81, 1966.

[15] M. R. C. McDowell and J. P. Coleman, Introduction to the Theory of Ion-Atom Collisions, North-Holland, Amsterdam, The Netherlands, 1970.

[16] D. S. F. Crothers, "Second-order continuum-distorted-wave double-scattering nlm transitions," Journal of Physics B, vol. 18, no. 14, pp. 2893-2906, 1985.

[17] G. J. N. Brown and D. S. F. Crothers, "Close-coupling symmetrized variational continuum distorted-wave theory: electron capture to excited states in $\mathrm{p}-\mathrm{H}$ collisions," Physical Review Letters, vol. 76, no. 3, pp. 392-395, 1996.

[18] D. S. F. Crothers, D. M. McSherry, S. F. C. O’Rourke, M. B. Shah, C. McGrath, and H. B. Gilbody, "Magnetically quantized continuum distorted waves," Physical Review Letters, vol. 88, no. 5, pp. 532011-532014, 2002.

[19] R. T. Pedlow, S. F. C. O'Rourke, and D. S. F. Crothers, "Fully differentialcross sections for $3.6 \mathrm{MeVu}{ }^{-1} \mathrm{Au}^{\mathrm{Zp}+}+\mathrm{He}$ collisions," Physical Review A, vol. 72, no. 6, Article ID 062719, 2005.

[20] R. T. Pedlow, "Four-Body Ionization of Helium using Continuum Distorted-Waves," Ph.D. thesis, Queen's University Belfast, Belfast, UK, 2005.

[21] D. S. F. Crothers, Relativistic Heavy-Particle Collisions Theory, Kluwer Academic/Plenum, New York, NY, USA, 2000.

[22] D. S. F. Crothers, Semiclassical Dynamics and Relaxation, vol. 47 of Springer Series on Atomic, Optical, and Plasma Physics, Springer, Berlin, Germany, 2008.

[23] P. G. Burke and W. T. Robb, “The R-Matrix Theory of Atomic Processes," Advances in Atomic, Molecular, and Optical Physics, vol. 11, p. 144, 1975.

[24] Wikipedia Density Functional Theory, GOOGLE, 2009.

[25] A. Messiah, Quantum Mechanics I, II, Dover, Dover, Del, USA, 1999.

[26] L. D. Faddeev, "Scattering Theory for a three-particle system," Soviet Physics JETP, vol. 12, p. 1014, 1961.

[27] C. Lovelace, "Practical theory of three-particle states. I. Nonrelativistic," Physical Review, vol. 135, no. 5, pp. B1225B1249, 1964.

[28] N. Mishima, et al., "A Generalized Lovelace Equation in the Relativistic Faddeev Formalism II," Progress of Theoretical Physics, vol. 39, p. 1569, 1968.
[29] L. R. Dodd and K. R. Greider, "Rigorous solution of threebody scattering processes in the distorted-wave formalism," Physical Review, vol. 146, no. 3, pp. 675-686, 1966.

[30] R. Gayet, "Charge exchange scattering amplitude to first order of a three body expansion," Journal of Physics B, vol. 5, no. 3, pp. 483-491, 1972.

[31] J. Macek and S. Alston, "Theory of electron capture from a hydrogenlike ion by a bare ion," Physical Review A, vol. 26, no. 1, pp. 250-270, 1982.

[32] D. S. F. Crothers and K. M. Dunseath, "Target continuum distorted-wave theory for capture of inner-shell electrons by fully stripped ions," Journal of Physics B, vol. 20, no. 16, pp. 4115-4128, 1987.

[33] D. Belkić, I. Mančev, and J. Hanssen, "Four-body methods for high-energy ion-atom collisions," Reviews of Modern Physics, vol. 80, no. 1, pp. 249-341, 2008.

[34] D. Belkić, R. Gayet, and A. Salin, "Electron capture in highenergy ion-atom collisions," Physics Reports, vol. 56, no. 6, pp. 279-369, 1979.

[35] D. Belkić, Principles of Quantum Scattering Theory, Taylor \& Francis, London, UK, 2003.

[36] D. Belkić, Quantum Theory of High-Energy Ion-Atom Collisions, CRC Press, Boca Raton, Fla, USA; Taylor and Francis, London, UK, 2009.

[37] D. Belkić, "Review of theories on double electron capture in fast ion-atom collisions," Journal of Mathematical Chemistry, vol. 47, no. 4, pp. 1-48, 2010. 

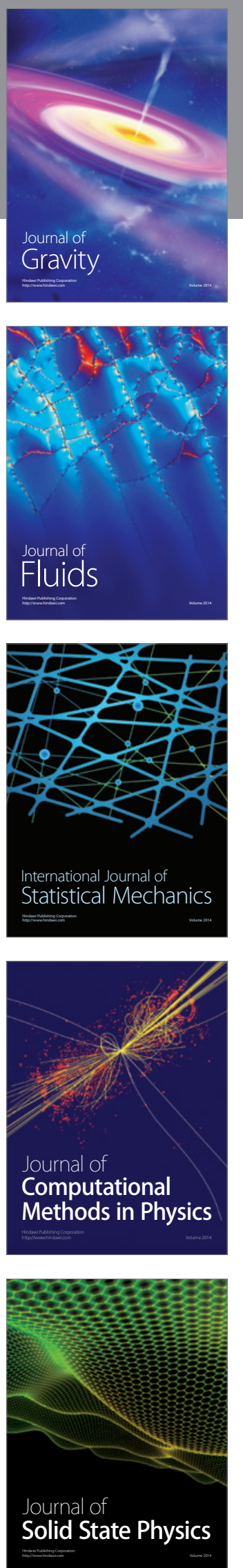

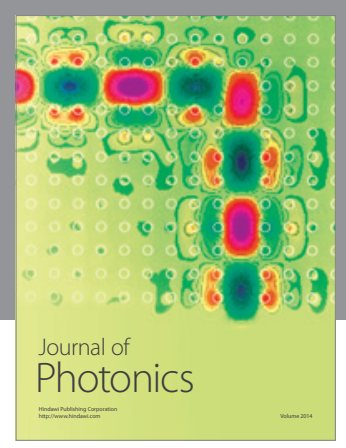

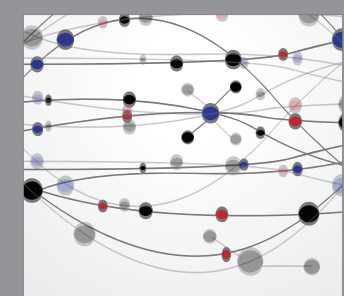

The Scientific World Journal
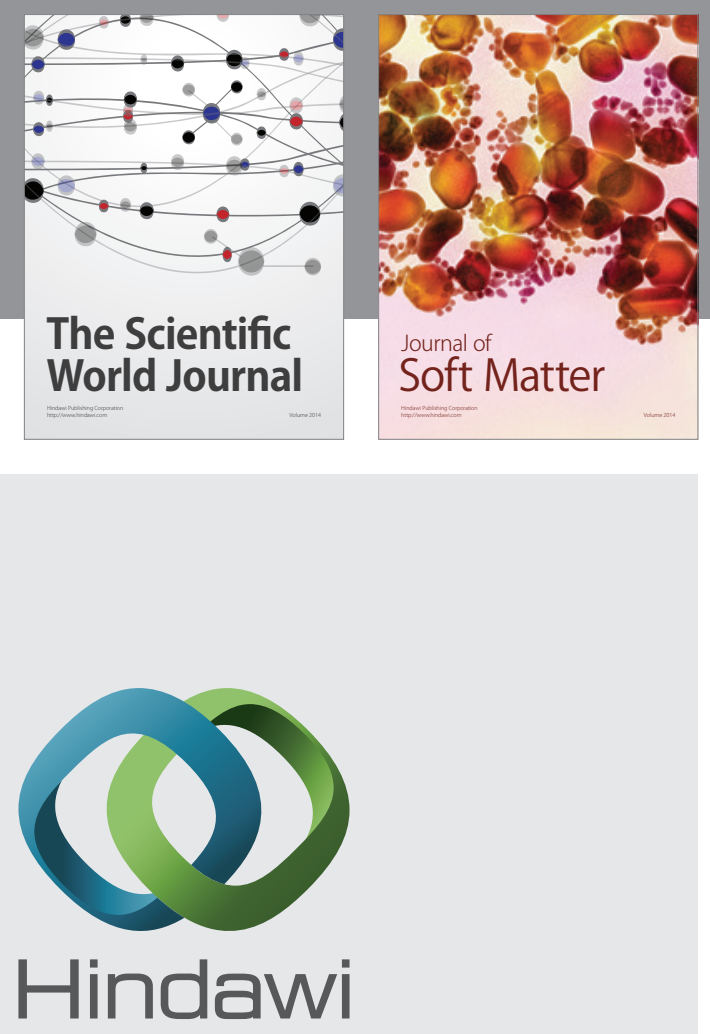

Submit your manuscripts at

http://www.hindawi.com
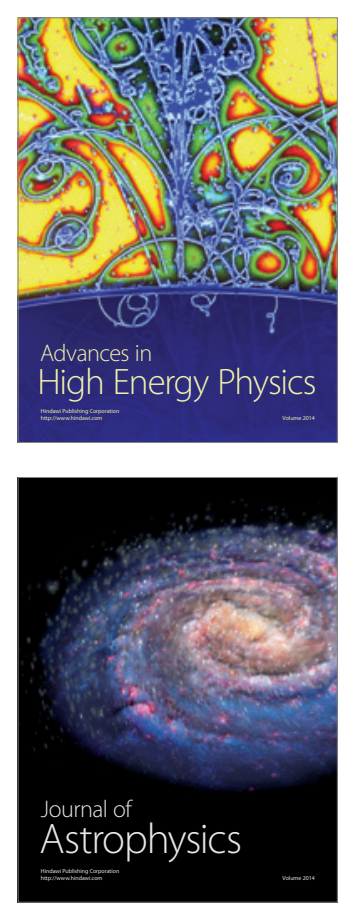
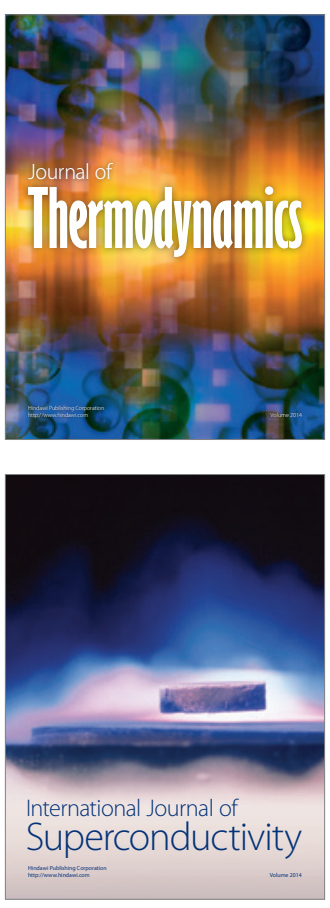
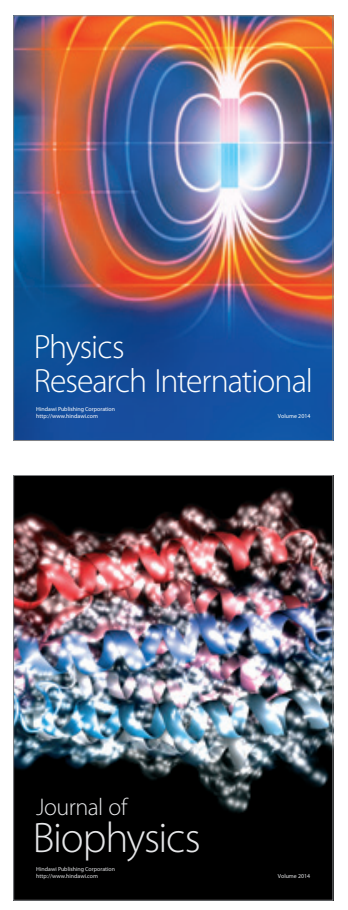
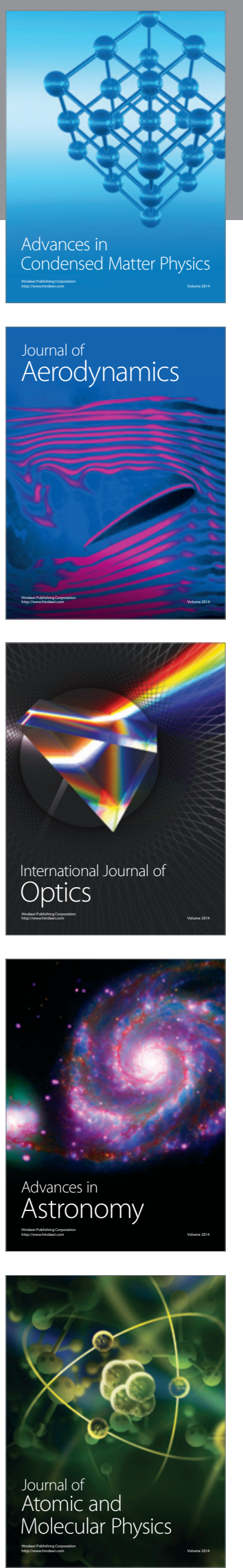